\title{
CHALLENGE AND SUPPORT FOR BREASTFEEDING IN HIGHLY MOTIVATED MALAYSIAN MOTHERS
}

\author{
Nazatul SB, Ruby H \\ Department of Physiology, Faculty of Medicine, Universiti Malaya, Kuala Lumpur
}

\begin{abstract}
:
The exclusive breastfeeding rate in Malaysia is very low. However in recent years the awareness of breastfeeding among mothers has increased. A preliminary qualitative research was carried out on these motivated mothers. The objective of this study was to understand the challenges encountered by breastfeeding mothers and to explore the support and motivation received by them. Information from the motivated mothers was obtained from focus group discussion. Some obstacles faced by the mothers were lack of knowledge on breastfeeding and lack of support from health professionals, parents and siblings. Facilities to express breast milk while at work were not readily available. The main motivation to breastfeed came from the mother herself and support from the husband. A holistic approach must be used to help mothers to continue breastfeeding. This includes breastfeeding promotion and education, setting up more Baby Friendly Hospitals, availability of breastfeeding support groups and provision of enough breastfeeding facilities at work and public places. (JUMMEC 2009; 12 (2): 70-73)
\end{abstract}

KEYWORDS: breastfeeding, challenges, motivation, promotion, support group

\section{Introduction}

Human lactation is the natural way to provide nutrition $(1,2)$ prevent childhood diseases $(3,4,5)$ and to provide love and affection to the baby $(6,7)$. Not only does breastfeeding benefit the baby, it also protects the mother from certain diseases $(8,9,10)$ and it is considered as a cost effective method of infant nutrition $(11,12)$. In addition, breastfeeding has always been the intended way of human survival.

Even though breastfeeding is the main method of infant nutrition, the exclusive breastfeeding (EBF) rate in Malaysia is very low. In a recent National Health and Morbidity Survey (NHMS III) held in 2006, only $14.5 \%$ of Malaysian mothers exclusively breastfed their babies up to 6-month old (13). On another note, the recent implementation of Baby Friendly Hospital Initiative has promoted the awareness of breastfeeding among mothers (14). We conducted a preliminary qualitative research on mothers who are highly motivated and who have decided to |breastfeed their baby before the baby was born. The objective of this study was to understand the challenges faced by breastfeeding mothers and to explore the support and motivation received by these mothers.

\section{Methodology}

The age of mothers ranged from 26 to 35 years old. The majority of them were multiparous. Most of them were professionals with total household income of more than RM4,000.00 per month. The majority obtained tertiary education. The main challenge faced by these mothers was the lack of breastfeeding knowledge. Even though they were aware of the benefits of breastfeeding, and they had intentions to breastfeed their babies, they were not given or did not seek out for enough information about breastfeeding techniques. These were illustrated by the following example:

".. not much of an exposure. Did not ask from anybody. Did not look for any information. I breastfed him at night but during daytime (when I was at work) he was given formula milk." (I,27, clerk)

\section{Correspondence:}

Nazatul Shiha binti Bachtiar

Department of Social and Preventive Medicine

Faculty of Medicine, Universiti Malaya

50603 Kuala Lumpur

Email: nazatulshiha@um.edu.my 
Most of these mothers faced the biggest challenge when they delivered their first child. The experience of handling a new born baby was overwhelming, let alone to breastfeed the baby. Several mothers also complained that they had some breastfeeding problems such as engorged breast, cracked nipple and inverted nipple. One mother said,

"First few days only a little bit of colostrum. By the third day the breast were engorged but nothing came out. My baby cried like mad. Very painful." (NS, 33, doctor)

These problems led to a stressful confinement period, as faced by some of the mothers. This was aggravated by the lack of support from their parents. In Malaysia, it is common for the parents or parents in law to take care of the new mother during the confinement. However, the strong belief held by the grandparents that formula milk is superior to breastmilk made the breastfeeding experience even more difficult as shown by these examples:

"...after that we stayed with my mother-in-law. If [it was] my own mother, we can say what we want. But if [it was] with my mother-in-law I couldn't say much. I didn't want to hurt her feelings. It was difficult to say... Furthermore my baby was their first grandson. During confinement, she used to take my baby and he slept in their room. They only gave him to me if he wanted to breastfeed..." ( $N, 26$, technician).

"...I started to breastfeed the brother [from a pair of twins]. I had to remind a lot of people. They wanted to give formula milk, I said "No I want to breastfeed him first..." $(F, 28$, lecturer).

Apart from challenges in the home, mothers also encountered difficulties in hospitals. Some of the hospitals, especially in the private sector do not have a policy in place to support breastfeeding. In some cases, no permission were asked from these mothers when formula milk was fed to the baby during the admission period.

"I delivered my first child at a non baby friendly hospital. They did not ask whether I want to breastfeed the child or not. Even the first day he was given a bottle of formula milk..." (A, 31, executive).

Some mothers had difficulty expressing their milk at the workplace. Problems such as no proper place to pump and non flexible working hours are the challenges faced by these mothers. Other issues that were discussed include postnatal blues, postnatal depression, breastfeeding discomfort, embarrassment, restricted freedom, insufficient milk, having a premature baby and having a pair of twins are part of the challenges in breastfeeding.

However, most of these mothers have succeeded in their mission to breastfeed their babies. The main motivation came from within themselves whereby they were determined to breastfeed their child. A mother mentioned,

"Starting from that [moment], when we went to the class we have decided... that whatever happened we wanted to breastfeed the baby. During the class the speaker had told us what would be the challenges... so we were told the expected problems beforehand..." (NK, 34, webmaster).

Apart from themselves, most of these highly motivated mothers were fully supported by their husbands.

"Surprising! When I was pregnant I wanted to breastfeed. We went to a shopping complex where a parenting expo was held. We were at the Medela booth. He said, 'This is a good brand.' He was more advanced! 'There is a website what, is it? Susuibu dot com?' He told me about the website. We bought the twin pump on the spot." (F, 28, lecturer).

"He will dismantle the breastpump apparatus, label all the bottles. Help like that is enough for me. He understands the breastfeeding process. When I wanted to breastfeed, he supports me." (I, 27, clerk).

"I was lucky that my husband supports me 100\%." (ZH, 31, technician).

Another important support received by these mothers were from the mother-to-mother support group. Even 
though no formal support group was mentioned, these mothers appreciated the help, information and moral support that they received from other breastfeeding mothers.

"..met with a relative who was a breastfeeding advocator. She told me about breastmilk expression. I had never imagined it before. Coincidently, my sisterin-law gave birth just 5 days before me. We motivated each other. She also breastfed her child..." (NK, 34, webmaster).

"At the office, I saw a friend expressing her milk. She told me how to do it. Only then [did] I understand a bit. After that, I bought a breastpump even though I was not even pregnant yet! I was motivated to breastfeed. My husband was surprised!" (I, 27, clerk).

Other factors that motivated and supported these mothers were bonding, good breastfeeding information from the hospital, supportive healthcare staff, supportive colleagues and flexible work time.

\section{Discussion}

Research conducted to explore the hindrances and motivation of breasfeeding in mothers from developing countries like Malaysia is scanty. Mothers from the present qualitative study were purposely recruited from a breastfeeding support group and they were highly motivated to breastfeed their babies. The rationale to select these mothers was to assess their perspectives and experience in managing breastfeeding in a typical Malaysian community. If these motivated mothers encountered difficulties in breastfeeding, what more for those who lack such motivation to breastfeed.

Therefore, the input from these motivated mothers are useful in looking at the breastfeeding problem, as a first step in exploring the root cause of non-motivation at the national level. Results showed that even though determination and motivation are important factors for successful breastfeeding, the mothers were not excluded from facing many challenges. The main challenges include inadequate breastfeeding knowledge, being a first time mother, breast and breastfeeding problems and lack of support from various parties.

\section{Conclusion}

A holistic approach must be used to help mothers to continue breastfeeding. This includes breastfeeding promotion and education to mothers and their immediate family members, setting up more Baby Friendly Hospitals covering the private hospitals, availability of breastfeeding support groups and provision of enough breastfeeding facilities at work and public places.

\section{References}

1. Jarjou LMA, Prentice A, et al. Randomized, placebocontrolled, calcium supplementation study in pregnant Gambian women: effects on breastmilk calcium concentrations and infant birth weight, growth, and bone mineral accretion in the first year of life, Am J Clin Nutr 2006; 83(3): 657-666.

2. Collins CT, Gibson RA, et al. Carbohydrate intake is the main determinant of growth in infants born $<33$ weeks' gestation when protein intake is adequate. Nutrition 2008; 24(5): 451-457.

3. Chantry CJ, Howard CR, et al. Full Breastfeeding Duration and Associated Decrease in Respiratory Tract Infection in US Children. Pediatrics 2006; 117(2): 425-432.

4. Bryan D-L, Hart PH, et al. Immunomodulatory constituents of human milk change in response to infant bronchiolitis. Pediatric Allergy and Immunology 2007; 18(6): 495-502.

5. Mihrshahi SI, Shuaib N, Oddy M, Ampon W. Prevalence of exclusive breastfeeding in Bangladesh and its association with diarrhoea and acute respiratory infection: Results of the Multiple Indicator Cluster Survey 2003. J of Health, Population and Nutrition 2007; 25(2): 195-204.

6. Gribble, K. Mental health, attachment and breastfeeding: implications for adopted children and their mothers. Int Breastfeeding J 2006; 1(1): 5.

7. Hammock EAD and Young LJ. Oxytocin, vasopressin and pair bonding: implications for autism. Philosophical Transactions of the Royal Society B: Biological Sciences 2006; 361(1476): 2187-2198. 
8. The Collaborative Group on Hormonal Factors in Breast. Breast cancer and breastfeeding: collaborative reanalysis of individual data from 47 epidemiological studies in 30 countries, including 50302 women with breast cancer and 96973 women without the disease. The Lancet 2002; 360(9328): 187-195.

9. Soon Young L, Miyong TK, et al. Effect of lifetime lactation on breast cancer risk: A Korean Women's Cohort Study. Intl J of Cancer 2003; 105(3): 390-393.

10. Chiaffarino F, Pelucchi $C$, et al. Breastfeeding and the risk of epithelial ovarian cancer in an Italian population. Gynecologic Oncology 2005; 98(2): 304308.

11. Weimer J. The Economic Benefits of Breastfeeding: A Review and Analysis. Food Assistance and Nutrition Research Report No. 13. Washington, DC: Food and Rural Economics Division, Economic Research Service, USDA; 2001.
12. Adriano $C$, Luca $R$, et al. Infant feeding and cost of health care: A cohort study. Acta Paediatrica 2006; 95(5): 540-546.

13. Salim F, Hassan Nudin SS, Muhammad Ismail HI, Aris T. Infant Feeding, in The Third National Health and Morbidity Survey 2006 (NHMS III). Kuala Lumpur: Ministry of Health Malaysia; 2007.

14. Merten S, Dratva J, Ackermann-Liebrich U. Do Baby-Friendly Hospitals Influence Breastfeeding Duration on a National Level? Pediatrics 2005; 116;702-708. 